\title{
Video Article \\ Assessing the Coherence of Parents' Short Narratives Regarding their Child Using the Five-Minute Speech Sample Procedure
}

\author{
Efrat Sher-Censor ${ }^{1}$ \\ ${ }^{1}$ The Interdisciplinary Graduate Program in Child Development and the Center for the Study of Child Development, University of Haifa \\ Correspondence to: Efrat Sher-Censor at esher@psy.haifa.ac.il \\ URL: https://www.jove.com/video/60025 \\ DOI: doi:10.3791/60025
}

Keywords: Behavior, Issue 151, Five minute speech sample, narratives, coherence, parent-child relationship, mother-child relationship, parental representations, close relationships, developmental psychology, FMSS-Coherence

Date Published: 9/19/2019

Citation: Sher-Censor, E. Assessing the Coherence of Parents' Short Narratives Regarding their Child Using the Five-Minute Speech Sample Procedure. J. Vis. Exp. (151), e60025, doi:10.3791/60025 (2019).

\section{Abstract}

Valid and efficient measures for assessing the quality of parent-child relationships are needed to facilitate research and evidence-based practice with parents. This paper focuses on such a method, namely Five-Minute Speech Sample-Coherence (FMSS-Coherence). In this method, a parent is asked to speak for five uninterrupted minutes about her/his child and their relationship. The resulted narrative is coded for coherence, namely the extent to which the parent provides in the narrative a clear, consistent, multidimensional and well-supported portrayal of the child. FMSS-Coherence is based on attachment research that shows that the coherence of parents' narratives is indicative of the quality of the parentchild relationship and child adjustment. It overcomes the limitations of attachment narrative measures which are typically labor intensive. FMSSCoherence is less nuanced than extant attachment narrative measures. Yet, studies of families from different cultural backgrounds, across different child ages and in the context of typically developing children as well as children with special needs suggest that coherence can be reliably evaluated using the FMSS procedure. Furthermore, parents' FMSS-Coherence is associated with parenting quality and children's socio-emotional adjustment. Thus, it holds promise for researchers and practitioners who seek a relatively time- and cost-effective method for assessing the coherence of parents' narratives regarding their child.

\section{Video Link}

The video component of this article can be found at https://www.jove.com/video/60025/

\section{Introduction}

Attachment theory suggests that the coherence of parents' narratives, namely the extent to which parents provide clear, consistent and multifaceted portrayals of their child, is of particular importance for learning about the emotional climate of the parent-child relationship ${ }^{1,2}$ According to this view, the coherence of parents' narratives captures the coherence of their internal representations (i.e., feelings, attributions and expectations) of the child. Parents' representations are defined as complex information processing rules that guide parents' interpretation of their child's behavior as well as parents' behavior toward the child ${ }^{1,3}$, and consequently shape child adjustment.

Some parents process information regarding their child in a flexible manner. Their representations of the child are presumably undistorted and include strengths as well as challenging aspects of the child's characteristic and the parent-child relationship. When asked to narrate regarding the child, these parents would presumably provide multifaceted and integrated (i.e., coherent ${ }^{4}$ ) descriptions of the child and their relationship. Importantly, parents' coherent representations are thought to facilitate an accurate interpretation of the child's signals as well as prompt and appropriate responses to the child (i.e., sensitive caregiving ${ }^{5}$ ). This in turn contributes to the child's sense of security and competence as well as better self-regulation ${ }^{6,7}$.

Other parents process information regarding their child in a distorted way, and construct incoherent representations, which could take different forms, such as unilateral representations that are negative and overemphasize the child's weaknesses, overwhelmed with concern, colored by difficulties in separateness from the child, idealizing or poorly integrated. Such representations are presumably mirrored in incoherent narratives that are unidimensional and/or inconsistent. Incoherent representations may hamper accurate interpretation of the child's signals and lead to parents' insensitive caregiving in terms of emotional detachment, hostility, rigidity and harshness, or overprotectiveness and intrusiveness ${ }^{1,2,4}$. The child in turn, might internalize the incoherent representations of the parent ${ }^{8}$, construct negative expectations regarding close relationships, learn to restrict or exaggerate emotional expressions in relational contexts ${ }^{9}$, and experience poorer socio-emotional adjustment ${ }^{10,11}$.

Attachment research supports to these notions ${ }^{7,9,10,11}$. Yet, extant methods to assess the coherence of parents' narratives involve labor intensive semi-structured interviews ${ }^{12}$. These include the Adult Attachment Interview ${ }^{13}$, in which parents are asked to narrate about their early attachment experiences, and the Working Model of the Child Interview ${ }^{14}$, the Parent Developmental Interview ${ }^{15}$ and the Insightful Assessment ${ }^{16,17}$, in which parents are invited to narrate regarding their child. Due to their length and costs, the employment of these methods in large-scale research and practice with parents is relatively limited. To overcome this drawback, this paper introduces a new method for assessing the coherence of parents' short narratives elicited using the FMSS procedure. 
The FMSS procedure was developed by the psychoanalyst and researcher Louis Gottschalk and his colleagues ${ }^{18,19}$. The researchers argued that although the procedure is brief, it maximizes the projection and expression of internal psychological states, response sets and attitudes as it requires narrating without the aid of prompts or responses from the interviewer ${ }^{18}$. Adult psychiatry researchers have embraced this procedure and have been using it to evaluate the quality of the relationships formed between mentally ill patients and their caregivers. In this context, a caregiver is invited to talk about the mentally ill relative and their relationship, and the FMSS is coded for expressed emotion (FMSS-EE ${ }^{20}$ ), namely caregivers' statements of criticism and emotional overinvolvement toward the relative. FMSS-EE is effective in indexing poor quality of caregiver-patient relationships and predicting patients' maladaptation and relapse risk ${ }^{21,22}$. Over the past three decades developmental and pediatric researchers have adopted the FMSS-EE method. The FMSS-EE coding was adapted to the context of parenting young children, for example, by adding the assessment of parents' positive comments regarding the child and the parent-child relationship (see references for examples ${ }^{23,24,25}$ ). Research on parents of children from infancy through adolescence have shown that FMSS-EE is related to parenting quality and is associated with and predicts the socio-emotional adjustment of the child ${ }^{26,27}$.

Attachment and FMSS-EE research have developed separately. Thus, EE research does not examine the coherence of the FMSS. To assess the coherence of parents' FMSS, the Insightful Assessment rating scales ${ }^{17}$ have been recently adapted to be used with the FMSS. This paper presents the protocol for collecting parents' FMSS and coding FMSS-Coherence. Results of studies that used the FMSS-Coherence method are described. Finally, directions for future research with FMSS-Coherence and issues that should be taken into consideration by researchers who plan to use FMSS-Coherence are discussed.

\section{Protocol}

The Human Research Review Board of the University of California Riverside, CA, USA and the University of Haifa, Israel, approved the procedures described here. This paper is an introduction to the FMSS procedure and its coherence coding. Using it requires further training provided by the author upon request.

\section{The FMSS procedure ${ }^{20}$}

This paper presents only the main steps of the FMSS procedure. Using the FMSS protocol requires its author's ${ }^{20}$ permission.

\section{Equipment}

1. Keep a voice recorder, stop-watch and word processing software ready.

\section{Interview procedure}

1. Sit with the parent in a quiet room. Administer the FMSS as the first measure to collect from the parent.

2. Turn on the audio recorder and ask the parent: "For the next 5 minutes, please tell me about your thoughts and feelings regarding your child. Please tell me what kind of a person your child is and how the two of you get along together." Turn on the stop-watch to count the 5 min.

3. If the parent falls silent for $30 \mathrm{~s}$ or longer, offer the following prompt (only once): "Please tell me anything about your child for a few more minutes."

\section{FMSS transcribing protocol}

1. Transcribe the FMSS, starting from the instructions given to the parent. This will enable coders to take into consideration interviewers' drifts from the protocol, which may affect the quality of the parental narrative.

2. Document in the transcript any of the following: changes in the parent's tone of voice (e.g., "laughs"; "changes tone of voice to mimic the child"); length of pauses in parent's speech (e.g., "20 seconds pause"); and external interruptions during the 5 min (e.g., "a phone rings for 10 seconds").

3. If the parent spoke for more than 5 min, mark when the 5 min ended.

4. Insert continuous line numbers to facilitate coders' consensus discussions.

\section{The FMSS-Coherence coding method}

NOTE: The complete manual that includes seven 7-point rating scales can be obtained from the author. Low and high scores of the scales and examples of coded FMSS are presented herein as an introduction to the coding system.

\section{Step 1: Rating six components of coherence}

1. For each of the following six subscales, use "+" or "-" signs throughout the transcribed FMSS to mark segments that reflect the subscales positively or negatively. Based on these "+" and "-" signs, determine a score for each subscale according to the following guidelines.

2. Focus:

1. Assign a high score when the parent refers to the child and/or to their relationship as the focal subject of the FMSS.

2. Assign a low score when the parent refers to other matters for the majority of the FMSS, or narrates about her/his children as a whole instead of focusing on the target child, e.g., "... I believe my schedule varies from the other jobs, so I usually work on Tuesday nights, Wednesday nights and Thursday nights, some weekends, but usually I get a Monday off and Friday off. I work two jobs, um 7:30 to $4: 30$ and the other one from 5:30 to about 8:30."

\section{Elaboration:}

1. Assign a high score when the parent provides detailed and rich descriptions of the child and/or the relationship. 
2. Assign a low score when the FMSS is laconic and includes only a few brief sentences, e.g., "He is a friendly boy. He is a good boy, there isn't anything special I could say about him. He takes an active part in the family and in school, he is a very good boy. He takes an active part in other settings as well. At home also. I don't know what else to say. This is it. This is his personality. There is nothing unusual about him."

4. Separateness:

1. Assign a high score when the parent describes the child as having a distinct personality with needs and desires that are different from her/his own.

2. Assign a low score when the parent shows difficulties in describing the child as a separate entity, e.g., "We are phenomenally close; we're very touchy feely mother. I kiss her I hold her I bathe with her. I'm proud that she's not afraid to show that she loves me anywhere anytime...she kisses me and holds me."

3. Assign a low score also when the parent shows boundary dissolution, namely, describes the parent's and child's roles as equal or reversed. For example, "I can consult with him more than with any of my close friends, even more than with my husband. I can ask him questions that are very personal. If I need something, I know I can go to him...he is not always available to me, but if I really need him, he is there for me."

5. Concern:

1. Assign a low score when the parent does not articulate worries or concerns.

2. Assign a high score when the parent refers several times to characteristics of the child or the relationship that concern her/him, e.g., "I am concerned about his social skills. I am worried that he doesn't have any close friends. I think it's affecting his selfconfidence. He keeps to himself a lot and I am worried about it. I don't know what to do, I just don't know what to do."

3. Reverse code the score given in Concern, as a low score is a positive marker of coherence.

6. Acceptance/rejection:

1. Assign a high score when the parent speaks lovingly of the child, is pleased and proud with the child's accomplishments, and displays empathy for the child's weaknesses and negative behaviors.

2. Assign a low score when the parent expresses rejection, disappointment, devaluation or a judgmental stance regarding the child, even if she/he also shows a few expressions of affection, e.g., "Um she's crazy (laughs). She is a little hyper. She cries when she doesn't get her way. She does very good in school, can do her homework on her own... but she is in everybody's business all the time and tries to be her teacher's pet."

7. Complexity:

1. Assign a high score when the parent addresses both positive and negative aspects of the child or the relationship in different contexts and provides supporting examples for most of her/his statements.

2. Assign a low score when the parent provides a unidimensional portrayal that focuses only on positive, or primarily negative or concerning aspects of the child and the relationship, or when the parent does not provide examples from everyday life to support her/his statements, e.g., "She is a charming girl, a very good girl. Compare to other children that I see around, she listens, she understands, she is very clever, she is polite. She is really a very good child. We have a strong relationship. That's it, I have nothing else to add."

\section{Step 2: Rating the coherence of the FMSS}

1. Read the FMSS again, mark contradictions in the text, and go over the scores assigned in step 1 to integrate impression of the FMSS as a whole.

2. Assign a high score when the FMSS was rated as high in focus, elaboration, separateness, concern (reverse scored), acceptance and complexity, and provided a consistent portrayal of the child and the relationship.

3. Assign a low-moderate score when the parent provided a description of the child or the relationship, but the FMSS was rated as low in focus, elaboration, separateness, concern (reverse scored), acceptance or complexity or if it included contradictory statements.

4. Assign a low score when the FMSS did not provide a description of the child or the relationship.

3. Step 3: Data analyses

NOTE: Depending on the research question, check if the continuous coherence 1-7 scores can be used or convert the scores into dichotomized coherent (score of 5 or higher) versus incoherent (score of 4 or lower) categories.

4. Coding example 1: A section of a transcribed FMSS of a mother of an 8-year-old boy.

1 "Ok, Joseph did very well last year at school. He got attendance awards and was one of the

2 highest students in his class in reading and got a silver medal. He's very friendly and outgoing.

$3 \mathrm{He}$ comes home from school and tells me that a new kid started, and that he made friends with

$4 \mathrm{him}$ and so um I'm very proud of him that he will go up and talk to other kids that don't know

5 anybody else. And at home he helps out he's, out of all my kids, he wants to help the most. He 6 likes to help clean and I'm just excited that he's very go getter and hopefully he stays that way.

$7 \mathrm{Umm}$ he fights with his brother a lot because they share a room and his brother likes to mess

8 up the room and of course Joseph feels responsible to clean it up because he's the older one.

9 and now he's starting to want to go and hang out with his friends. He's got a friend that lives

10 across the street and he goes over there a lot and he'll spend the night a couple times a week

11 um this summer and um I don't know. He's just becoming more confident and very outgoing

12 which is so exciting cause he used to be so shy and didn't want to talk to anybody before.

13 (30 second pause) Please tell me anything about Joseph just for a few more minutes.

$14 \mathrm{Um}$ he has been helping his dad out in the garden. We have like a garden in the backyard and

15 he likes to go out and help pick vegetables, pull the weeds and help water."

1. Assign a high score in Focus, as the mother talks only about the child (no "-" signs). 
2. For Elaboration, denote a "+" next to the following: doing well in school (lines 1-2); very friendly and outgoing (lines 3-5), helps (lines 5-6) fights with sibling (lines 7-8), hangs out with friends (lines 9-11), helps dad (lines 14-15). Accordingly, assign a high score in Elaboration.

3. Assign a high score in Separateness, as there are no separateness issues (no "-" signs).

4. Assign a low score in Concern, as the mother does not articulate any worries or concerns (no "-" signs), and reverse code the score from low to high.

5. For Acceptance, denote a "+" near the following: the mother is proud (line 4), excited to see improvement in the child's behavior (line 12), when relating to a negative behavior she expresses an understanding (lines 7-8). Accordingly, assign a high score in Acceptance.

6. Assign a high score in Complexity, as the mother describes different child's characteristics (school performance, relationships with peers and family), relates to his strength (did well in school, friendly and outgoing, helps) and weaknesses (fights with sibling, used to be shy) and supports her statements with examples.

7. Rate the FMSS as high in Coherence, based on the scores given in the above subscales as well as lack of contradictions in the text.

5. Coding example 2: A section of a transcribed FMSS of a mother of a 4-year-old boy diagnosed with Autism Spectrum Disorder (ASD).

1 "James is a loveable child, not only I love him, his father and his sister also love him very much.

2 He used to get upset easily, perhaps it's because it was hard for him to express his emotions

3 and thoughts, but now he speaks better, in short sentences, but it is easier now to understand

$4 \mathrm{him}$. His relationship with his sister and cousins has also improved. He loves to play with them.

5 When he was younger it was difficult because he did not know how to play and ended up alone.

6 But now he succeeds more, which is great. I noticed that it helps him if we explain to him in

7 advance what's going to happen. For example, if I want to go with him to the zoo, I will talk

8 with him about it before we leave home. If I don't prepare him and try to drive in a route that

9 is not the one that he takes every day, he will start screaming. It's not that everything is easy,

10 for example, yesterday I returned home very tired from work. He wanted something but I just

11 couldn't figure out what he wanted. We tried to talk, but we just couldn't communicate. He

12 became upset and screamed and cried and I was also very upset, and he probably noticed it.

13 I hugged him and kissed him until he calmed down and then I told him let's try again, and this

14 time we succeeded. I understood what he wanted. So there are moments in which we still

15 experience difficulties but I see improvement all the time. What else? He likes to play with

16 other children, he is good in "catch" games. For example, last weekend we visited friends and

17 he played "catch" with their daughter so nicely. We really enjoyed watching them. So more

18 and more there are moments in which I say to myself that he is growing to be an easy child."

1. Assign a high score in Focus, as the mother narrates solely on the target child (no "-" signs).

2. For Elaboration, denote a "+" next to the detailed examples of the following: there is improvement in child's behavior (lines 3-4, 6), they still experience some difficulties (lines 10-14); and the child is good in "catch" (lines 16-17). Accordingly, assign a high score in Elaboration.

3. Assign a high score in Separateness, as there are no indicators of difficulties in separateness and the mother describes the child as a separate person, with needs and desires that are different from her own (no "-" signs).

4. Assign a low score in Concern, as no "-" signs are denoted. The mother is not overwhelmed with concern. She describes the child difficulties, which are probably related to his ASD diagnosis, but emphasizes the improvements she sees in the child's behavior and their relationship (lines $3-4,6,15,18$ ), and communicates a sense of competence in parenting the child and helping him acquire more adaptive behaviors (lines $6-7,13-14$ ).

5. Reverse the concern score from low to high.

6. For Acceptance, denote a "+" near the following: the mother's expression of warmth (lines 1,13,18), enjoyment in the child's strength and development (lines 6,17), and expression of empathy and understanding when describing the child's negative behaviors (lines 2 , $6-8,13$ ). Accordingly, and as there are no sign of rejection (no "-" signs), assign a high score in Acceptance.

7. Assign a high score in Complexity, as the mother addresses different aspects of the child (language, emotional, social, behavioral and motor skills), narrates about the child's relationships with varied people and describes his behavior in different contexts (at home, on the way to the zoo, at their friends' house), describes the child's strengths (lines 4, 15-17) as well as weaknesses (lines 2, 9-12) and provides vivid examples from everyday life to support her descriptions (lines 3-4, 6, 10-14, 16-17).

8. Assign a high score Coherence, in light of the above scores in the coherence sub-scales and as the mother provides a clear, consistent and multifaceted portrayal of the child and their relationship that is not unduly colored by the child's ASD and behavior problems.

6. Coding example 3: A section of a transcribed FMSS of a mother of a 16 year-old-girl.

1 "She is my first child, so every step in her development was new to me and maybe because of

2 that, everything is so dramatic with her, or maybe l'm just overreacting. For example, the

3 terrible-twos was terrible (laughs) she resisted all the time and had tantrum attacks but only

4 in retrospect I realized that I reacted in a wrong way, and every time it's the same story, only

5 in retrospect I understand where I was wrong. Now she is in her adolescence

6 years, and it's difficult for me. She is stubborn, she disobeys me, and this annoys me.

7 Her room is always a mess. When I go to work, I miss her but when I come home, we just

8 don't get along. She thinks only of how to go out and have fun with her friends.

9 She does not study hard enough. I know she can do better but she does not care.

10 The message I get from her is "you're old and don't understand anything".

11 It really gets me angry."

1. Assign a high score in Focus, as the mother talks only about the child (no "-" signs).

2. For Elaboration, denote a "+" next to the description of the child's behavior at age 2 (line 3 ) and the examples of the difficulties the mother is currently experiencing with her daughter (lines 6-10). Accordingly, assign a high score in Elaboration. 
3. Assign a high score in Separateness, as the mother describes her daughter as having a distinct personality (no "-" signs).

4. For Concern, denote a "+" next to the description of the concern the mother experiences regarding her parenting (lines 2-5). In light of these remarks, assign a high score in Concern.

5. Reverse the score in Concern from high to low.

6. For Acceptance, denote a "-" next to the mother's expressions of rejection of her daughter when stating that everything is dramatic with the child (line 2) and listing the daughter's behaviors that annoy and make the mother angry (lines 6-11). Accordingly, assign a low score in Acceptance.

7. Assign a low score in Complexity as the narrative is one-sided negative and relates solely to aspects that concern (lines 2-5) or upset (lines 2, 6-11) the mother.

8. Assign a low score in Coherence, as although the narrative is consistent, it is one-sided negative, and colored by the mother's concern and negative view of her child.

7. Establishing reliability

1. Before coding FMSS transcripts from the study, establish inter-rater reliability with the FMSS-Coherence authors.

2. Code and have another trained coder code at least $20 \%$ of the narratives of the sample to establish inter-coder reliability ${ }^{30}$ NOTE: With small samples, it is recommended that a higher percentage of the FMSS would be coded by the two coders ${ }^{30}$.

3. Only after establishing reliability, discuss discrepancies in the scores and resolve them through consensus.

4. Calculate interrater reliability using Intraclass Correlation Coefficient (ICC) for the continuous coherence scores and Kappa for the dichotomized coherence scores ${ }^{30}$.

\section{Representative Results}

Interrater reliability

Interrater reliability of the FMSS-Coherence scores ranged from very good to excellent across prior studies. ICC of the continuous scores ranged between 0.86 and $0.95^{29,30}$, and Kappa ranged between 0.83 and $1.00^{31,33}$.

\section{FMSS-Coherence, observed parenting and child adjustment}

In support of the notion that parents' coherent narratives are associated with more sensitive caregiving, a study of mothers and young children with ASD attending special education schools indicated that mothers who provided coherent FMSS showed higher emotional availability during observed play interaction with their child as compared with mothers who provided incoherent FMSS. In other words, mothers who provided coherent FMSS were more sensitive, provided more appropriate structuring, and were less intrusive when interacting with their child ${ }^{31}$ (see Figure 1).

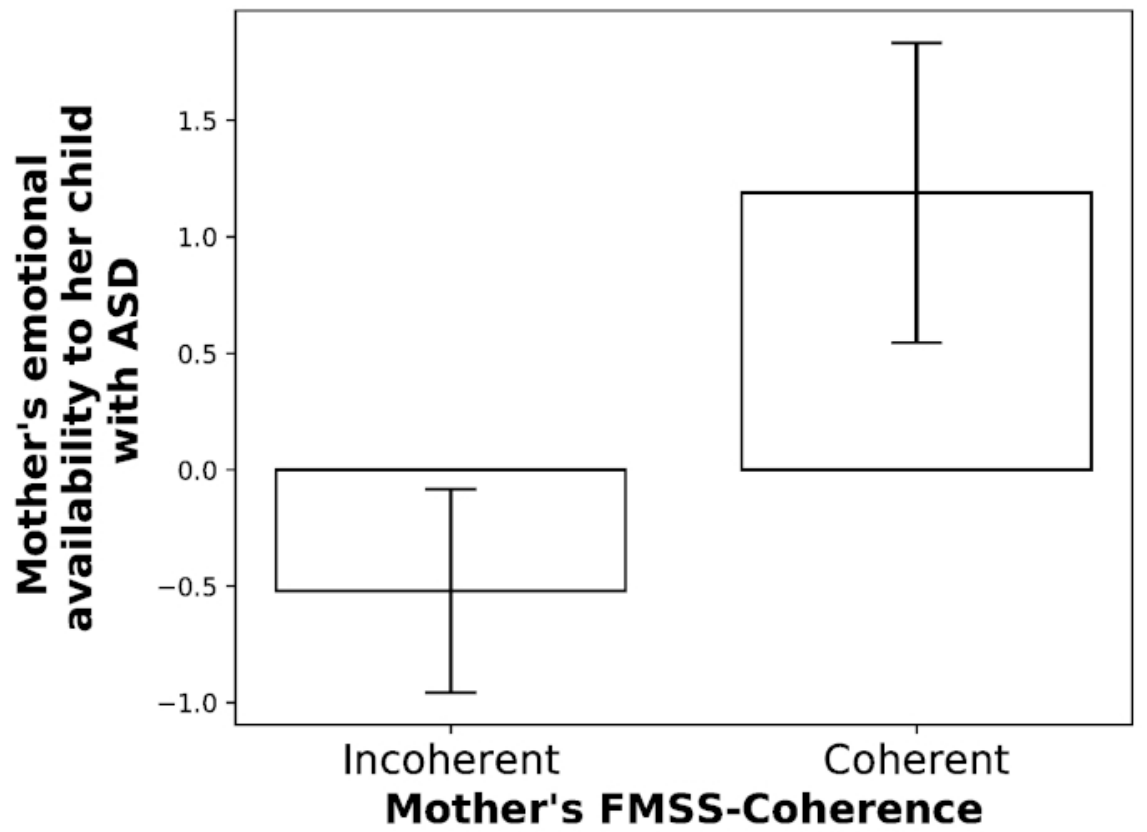

Figure 1: Mothers' mean emotional availability to their child with ASD by mothers' FMSS-Coherence. Error bars represent standard errors of the mean $(p<0.05)$. Please click here to view a larger version of this figure. 
In line with the notion offered by attachment researchers that parents' representations are internalized by their children, preschool children whose mothers provided incoherent FMSS showed more negative depictions of the parent-child relationship in their play-narratives than children whose mothers provided coherent FMSS ${ }^{29}$. Finally, as expected, parents' coherent FMSS are related to better socio-emotional adjustment of their children. The coherence of mothers' FMSS is associated with fewer behavioral problems of their toddlers ${ }^{30}$, preschoolers ${ }^{32}$ and school-aged children (Davidovitch, M., 2018). In addition, mothers' FMSS-Coherence predicts a decrease in externalizing behavior problems, an increase in ego resiliency, and an increase in peer acceptance from preschool to first grade among children with self-regulation difficulties ${ }^{33}$.

\section{Applying the FMSS-Coherence method to other relational contexts}

Recent findings point to the utility of FMSS-Coherence in research of other close relationships. A study in which pregnant mothers provided FMSS regarding the father-to-be found that lower coherence of the FMSS was associated with mothers' childhood maltreatment experiences, and this link was mediated by mothers' post-traumatic stress disorder (PTSD) symptoms (River, L., Castillo, M., Narayan, A., Sher-Censor, E., Lieberman, A., 2019). In another study in which adolescents provided FMSS regarding their mothers, adolescents' coherent FMSS mediated the association between higher maternal sensitive guidance, observed during mother-adolescent dialogue on autobiographic emotional events, and fewer behavior problems of the adolescents, as reported by adolescents' examiners ${ }^{34}$. This result is in line with attachment theory notion that when the child receives sensitive caregiving, she/he would construct coherent representations of the relationship with the caregiver, which in turn would facilitate better adjustment of the child ${ }^{8,9,10,11}$.

\section{FMSS-Coherence and participants' characteristics}

To support the notion that FMSS-Coherence captures quality of the relationship, FMSS-Coherence studies controlled for several participants' characteristics. These included mother and child age ${ }^{29,30,31,32,33}$, birth order ${ }^{29,30,31,32,33}$, child gender ${ }^{29,30,31,32,33}$, marital status ${ }^{29,32,33}$, mothers' and adolescents' receptive vocabulary and/or education level ${ }^{29,30,31,32,33}$, family socioeconomic status ${ }^{29,30,32,33}$, maternal psychological distress, economic distress and health condition ${ }^{31}$, and adaptive behavior of children with ASD $^{31}$. Of these characteristics, only mothers' receptive vocabulary and education level were significantly related to mothers' FMSS-Coherence and adolescent gender was related to adolescents' FMSS-Coherence, with girls showing higher levels of coherence than boys. Notably, the associations of FMSS-Coherence with observed parenting and child socio-emotional adjustment were significant when controlling for these characteristics ${ }^{30,31,32,33}$. Finally, studies suggest that FMSS-Coherence is valid among ethnic minorities and immigrant families (in the US: Hispanic and Black ${ }^{32}$; in Israel: Arab-Israelis ${ }^{31}$; and immigrants from the Former Soviet Union and Western countries ${ }^{34}$ ).

\section{Discussion}

Four of our previous studies examined both the coherence and EE in parents' FMSS (Davidovitch, M., 2018) ${ }^{29,30,32}$. Separate coding teams rated FMSS-Coherence and FMSS-EE. Three of these studies showed that FMSS-Coherence and the criticism component of FMSS-EE were significantly negatively correlated, although the associations were small to moderate (Davidovitch, M., 2018) ${ }^{29,32}$. The fourth study did not find a significant association between FMSS-Coherence and EE-criticism ${ }^{30}$. In addition, the four studies showed that FMSS-Coherence is not significantly associated with other components of FMSS-EE, namely, emotional overinvolvement and positive comments. Furthermore, FMSS-Coherence had a unique contribution to the explained variance of child adjustment indices. In one study, FMSS-EE was associated with mothers' reports of preschoolers' behavior problems while FMSS-Coherence was associated with observers' reports of the preschoolers' behavior problems ${ }^{32}$. In another study, only FMSS-Coherence and not FMSS-EE was associated with preschoolers' descriptions of parents in play narratives ${ }^{29}$; Finally, in two studies FMSS-Coherence contributed to the explained variance of toddlers' ${ }^{30}$ and school aged children's (Davidovitch, M., 2018) externalizing behavior problems, as reported by the mother, above and beyond the contribution of FMSS-EE. Taken together, these results suggest that FMSS-Coherence is distinct from FMSS-EE and point to the added value of assessing the coherence of parents' FMSS in relation to the traditional EE coding.

Researchers who wish to use FMSS-Coherence should take into consideration the following. Administrating the FMSS as the first measure of the research procedure is vital, as any measure administered prior to the FMSS may color parents' narratives. It is also important that the parent will be alone in the room with the interviewer, without the presence of other family members, to allow the parent to speak freely about the child.

Minimal interaction between the parent and the interviewer during the FMSS is critical. FMSS research suggests that the vast majority of parents are able to complete the task of speaking uninterruptedly about their child for $5 \mathrm{~min}$. Yet, researchers of high risk families, such as homeless parents ${ }^{35}$ and mothers of twins who gave birth before the age of $20^{23}$ suggest to provide a series of standardized prompts when parents experience difficulties in narrating for $5 \mathrm{~min}$ (e.g., "In what ways would you like your child to be different?"; "What is your child like in comparison with other children the same age?" ${ }^{23}$ ). These studies examined only FMSS-EE. As such prompts explicitly invite the parent to narrate about varied aspects of the child's characteristics, it may lead to higher scores in the elaboration and complexity components of coherence and influence the final coherence score. Research is required to compare results obtained with and without additional prompts. Such studies conducted with high- and low-risk families may indicate whether there are benefits in using such modified protocol for evaluating parents' FMSS-Coherence, and if so, in which contexts.

Finally, coding the six subscales (focus, elaboration, separateness, concern, acceptance/rejection and complexity) before rating the final coherence score is warranted to improve coding quality. To date, studies have looked only at the final coherence score. Nevertheless, future research with large-scale samples may examine whether the profile of strengths and difficulties parents are showing in their FMSS, as reflected in their scores on the six subscales, are related to distinct parenting behaviors and child outcomes (e.g., parents who provide a meager FMSS versus parents who show a high level of concern). Such an approach may be particularity useful in clinical settings, as it may facilitate tailoring individualized interventions that meet the specific difficulties parents expressed in their $\mathrm{FMSS}^{36}$.

FMSS-Coherence is a promising method for research of the parent-child relationship and other close relationships. Nevertheless, two limitations should be noted. First, validation of the method in countries other than the United States and Israel is required. Second, as the FMSS includes only a single broad question, it can be easily adapted to different contexts. As shown above, FMSS-Coherence can be used not only for interviewing parents about their children, but also for interviewing them about their romantic partner (River, L., et al., 2019) and for interviewing adolescents about their parents ${ }^{34}$. However, extending the use of the FMSS-Coherence method to other relational contexts should be done with caution. For example, FMSS-Coherence may be less applicable for assessing the quality of relationships that do not involve caregiving. In 
addition, prior EE research documented the validity of the FMSS-EE and the TMSS-EE (i.e., Three-Minute Speech Sample-EE) for children aged 6 years and up, who were asked to narrate about their parents ${ }^{37}$ or siblings ${ }^{38}$. Narrative skills, and abstract and critical thinking, which are vital for narrating coherently, develop significantly during the transition from childhood to adolescence ${ }^{39,40}$. Thus, the FMSS-Coherence method may not be applicable to children younger than mid-adolescence.

Additional research is needed to address the following issues. The first future direction involves examining whether parents' FMSS-Coherence is associated with parents' sensitivity and child attachment, the main outcomes of parents' narrative coherence examined in attachment research that used lengthy interview protocols ${ }^{12,14,15,16}$. Support for the link between FMSS-Coherence and mothers' sensitivity comes from the study described above of mothers of children diagnosed with $A_{S D}{ }^{31}$. Although the results of this study are promising, replication is needed with mothers of typically developing children. Second, although varied participants' characteristics were examined in prior FMSS-Coherence research $^{29,30,31,32,33,34}$, whether personality characteristics of parents shape the coherence of their FMSS awaits future research. Third, future studies would benefit from examining not only mothers' but also fathers' FMSS-Coherence and their association with paternal sensitivity and child socio-emotional adaptation. Fourth, most studies used the dichotomized coherent versus incoherent categories (e.g. ${ }^{31,32,33}$ ). This is in line with attachment research that typically uses a two-step coding procedure that begins with continuous scales and ends with classifying parents into categories that are used in data analyse ${ }^{12,13,14,15,16}$. The notion underlying this approach is that coherent narratives and incoherent narratives are qualitatively different ${ }^{4}$. However, growing evidence from attachment research ${ }^{41}$ as well as a few FMSS-Coherence studies ${ }^{30,34}$ suggest that the variation underlying FMSS-Coherence may be distributed continuously. Thus, additional studies are needed with large samples that would allow examination of individual differences among parents who narrate coherently and among parents who narrate incoherently and their associations with parenting quality and child's outcomes.

\section{Disclosures}

The author has nothing to disclose.

\section{Acknowledgments}

This research was supported by the National Institute of Health Grant 1R03HD065036-01A and the National Science Foundation Grant 1628820 awarded to Tuppett M. Yates and Efrat Sher-Censor and the Herta and Paul Amir Faculty of Social Science, the University of Haifa, Israel. The author would like to thank Dr. Smadar Dolev for taking part in the film.

\section{References}

1. Bowlby, J. Attachment and loss: Vol. 1. Attachment. New York: Basic Books (1982)

2. Bretherton, I. Communication patterns, internal working models, and the intergenerational transmission of attachment relationships. Infant Mental Health Journal. 11, 237-252. (1990).

3. Main, M., Kaplan, N., Cassidy, J. Security in infancy, childhood and adulthood: A move to the level of representations. Monographs of the Society for Research in Child Development. 50 (1-2, Serial No. 209), 66-104. (1985).

4. Oppenheim, D. Child, parent, and parent-child emotion narratives: Implications for developmental psychopathology. Developmental Psychopathology. 18, 771-790. (2006).

5. Ainsworth, M. D. S., Bell, S. M., Stayton, D. J. Infant-mother attachment and social development. In M. P. Richards (Ed.), The introduction of the child into a social world. (pp. 99-135). London: Cambridge University Press. (1974).

6. Dykas, M. J., Cassidy, J. Attachment and the processing of social information across the life span: Theory and evidence. Psychological Bulletin. 137 (1), 19-46. (2011).

7. Sroufe, L. A. Attachment and development: A prospective, longitudinal study from birth to adulthood. Attachment and Human Development. 7 (4), 349-367. (2005).

8. Fonagy, P., Target, M. Attachment and reflective function: Their role in self-organization. Developmental Psychopathology. 9, 679-700. (1997).

9. Cassidy, J. Emotion regulation: Influences of attachment relationships. Monographs of the Society for Research in Child Development. 59 228-249. (1994).

10. Oppenheim, D., Goldsmith, D., Koren-Karie, N. Maternal insightfulness and preschoolers' emotion and behavior problems: Reciprocal influences in a day treatment program. Infant Mental Health Journal. 25, 352-367. (2004).

11. Rosenblum, K. L., McDonough, S., Muzik, M., Miller, A., Sameroff, A. Maternal representations of the infant: Associations with infant response to the still face. Child Development. 73, 999-1015. (2002).

12. Vreeswijk, C. M., Maas, A. J. B., van Bakel, H. J. Parental representations: A systematic review of the working model of the child interview. Infant Mental Health Journal. 33 (3), 314-328. (2012).

13. Main, M., Hesse, E., Goldwyn, R. Studying differences in language usage in recounting attachment history: An introduction to the AAI. In H. Steele \& M. Steele (Eds.), Clinical applications of the Adult Attachment Interview. (pp. 31-68). New York: Guilford Press (2008).

14. Zeanah, C.H., Benoit, D., Hirshberg, L., Barton, M.L., Regan, C. Mothers' representations of their infants are concordant with infant attachment classifications. Developmental Issues in Psychiatry and Psychology. 1, 9-18 (1994).

15. Aber, J. L., Belsky, J., Slade, A., Crnic, K. Stability and change in mothers' representations of their relationship with their toddlers. Developmental Psychology. 35 (4), 1038-1047. (1999).

16. Koren-Karie, N., Oppenheim, D., Dolev, S., Sher, E., Etzion-Carasso, A. Mothers' insightfulness regarding their infants' internal experience: Relations with maternal sensitivity and infant attachment. Developmental Psychology., 38, 534-542. (2002).

17. Koren-Karie, N., Oppenheim, D. Parental insightfulness: Retrospect and prospect. Attachment \& Human Development. 20, 223-236. (2018).

18. Gottschalk, L. A., Gleser, G. C. The measurement of psychological states through the content analysis of verbal behavior. Berkeley: University of California Press. (1969).

19. Gottschalk, L. A., Gleser, G. C., Daniels, R. S., Block, S. The speech patterns of schizophrenic patients: A method of assessing relative degree of personal disorganization and social alienation. Journal of Nervous and Mental Disease. 127 (2), 153-166 (1958). 
20. Magaňa, A. B., et al. A brief method for assessing expressed emotion in relatives of psychiatric patients. Psychiatry Research. 17, $203-212$. (1986).

21. Barrowclough, C., Hooley, J. M. Attributions and expressed emotion: A review. Clinical Psychology Review. 23, 849-880. (2003).

22. Hooley, J. M. Expressed Emotion and relapse of psychopathology. Annual Review of Clinical Psychology. 3, 329-352. (2007).

23. Caspi, A., et al., Maternal Expressed Emotion predicts children's antisocial behavior problems: Using monozygotic-twin differences to identify environmental effects on behavioral development. Developmental Psychology. 40, 149-161. (2004).

24. Daley, D., Sonuga-Barke, E. J. S., Thompson, M. Assessing expressed emotion in mothers of preschool AD/HD children: Psychometric properties of a modified speech sample. British Journal of Clinical Psychology. 42, 53-67. (2003).

25. Pasalich, D. S., Dadds, M. R., Hawes, D. J., Brennan, J. Assessing relational schemas in parents of children with externalizing behavior disorders: Reliability and validity of the Family Affective Attitude Rating Scale. Psychiatry Research. 185, 438-443. (2011).

26. Sher-Censor, E. Five Minute Speech Sample in developmental research: A review. Developmental Review. 36, 127-155. (2015).

27. Weston, S., Hawes, D. J., Pasalich, D. S. The five minute speech sample as a measure of parent-child dynamics: Evidence from observational research. Journal of Child and Family Studies. 26, 118-136. (2017).

28. Syed, M., Nelson, S. C. Guidelines for establishing reliability when coding narrative data. Emerging Adulthood. 3, 375-387. (2015).

29. Sher-Censor, E., Grey, K., Yates, M. T. The intergenerational congruence of narrative affective content and narrative coherence. International Journal of Behavioral Development. 37, 340-348. (2013).

30. Sher-Censor, E., Shulman, C., Cohen, E. Associations among mothers' representations of their relationship with their toddlers, maternal parenting stress, and toddlers' internalizing and externalizing behaviors. Infant Behavior and Development. 50, 132-139. (2018).

31. Sher-Censor, E., Dolev, S., Said, M., Baransi, N., Amara, K. Coherence of representations regarding the child, resolution of the child's diagnosis and emotional availability: A study of Arab-Israeli mothers of children with ASD. Journal of Autism and Developmental Disorders. 47, 3139-3149. (2017).

32. Sher-Censor, E. Yates, M. T. Mothers' Expressed Emotion and narrative coherence: Associations with preschoolers' behavior problems in a multiethnic sample. Journal of Child and Family Studies. 24, 1392-1405. (2015).

33. Sher-Censor, E., Khafi, T., Yates, T. M. Preschoolers' self-regulation moderates relations between mothers' representations and children's adjustment to school. Developmental Psychology. 52, 1793-1804. (2016).

34. Sher-Censor, E., Koren-Karie, N., Getzov, S. Rotman, P. Mother- adolescent dialogues and adolescents' behavior problems in a multicultural sample: The mediating role of representations. Journal of Research on Adolescence. 28, 211-228. (2017).

35. Narayan, A. J., Sapienza, J. K., Monn, A. R., Lingras, K. A., Masten, A. S. Risk, vulnerability, and protective processes of parental expressed emotion for children's peer relationships in contexts of parental violence. Journal of Clinical Child and Adolescent Psychology. 44 (4), 676-688. (2015).

36. Dozier, M., Sepulveda, S. Foster mother state of mind and treatment use: Different challenges for different people. Infant Mental Health Journal. 25, 368-378 (2004).

37. Marshall, V. G., Longwell, L., Goldstein, M. J., Swanson, J. M. Family factors associated with aggressive symptomatology in boys with Attention Deficit Hyperactivity Disorder: A research note. Journal of Child Psychology and Psychiatry. 31, 629-636. (1990).

38. Yelland, I., Daley, D. Expressed emotion in children: Associations with sibling relationships. Child: Care, Health and Development., 35, 568-577 (2009).

39. Habermas, T., Bluck, S. Getting a life: The emergence of the life story in adolescence. Psychological Bulletin., 126, 748-769. (2000).

40. McLean, K. C., Thorne, A. Late adolescents' self-defining memories about relationships. Developmental Psychology. 39, 635-645. (2003).

41. Roisman, G. I., Fraley, R. C., Belsky, J. A taxometric study of the Adult Attachment Interview. Developmental Psychology. 43, 675. (2007). 